IMA Journal of Applied Mathematics (2014) 79, 1126-1138

doi:10.1093/imamat/hxt010

Advance Access publication on 24 February 2013

\title{
Real-time active wave control with preservation of wanted field
}

\author{
SERGEY V. UtYUZHNiKov \\ School of Mechanical, Aerospace and Civil Engineering, University of Manchester, Manchester \\ M13 9PL, UK \\ s.utyuzhnikov@manchester.ac.uk
}

[Received on 21 November 2011; revised on 1 October 2012; accepted on 1 February 2013]

\begin{abstract}
In active wave control, a quite arbitrary bounded domain is protected from the field (noise), generated outside, via implementation of additional sources. The secondary sources can be situated at the boundary of the domain to be shielded. Apart from the noise, the existence of the required field, generated inside the protected domain, is also allowed. The solution to the problem is obtained in a rather general formulation. The input information to the secondary sources is based on the total field at the perimeter of the shielded domain only. In the previous publications, the input data for the control were supposed to be the total field from the primary sources. It can be proven that such a control doubles wanted sound outside the protected domain. In contrast to the previous studies, the reverse effect of the secondary sources on the input data (due to wanted sound) is taken into account. This makes the obtained solution to be more realistic for practical applications. Some limitations of the proposed approach are also discussed.
\end{abstract}

Keywords: real-time; active control; active noise shielding; surface potentials; difference potential method; non-stationary problem.

\section{Introduction}

We assume that in the active control (AC) problem, some domain, which is usually bounded, is protected from the unwanted field coming from outside. The sources of this field situated outside the shielded domain are considered as noise sources. In turn, all possible internal sources are interpreted as the wanted ones. The noise attenuation in the protected domain is realized via implementation of additional (secondary) sources situated outside the domain to be shielded. Practically, they are often distributed on the perimeter of this domain. In contrast to passive control methods, there is no mechanical insulation. It is to be noted that the passive and control methods often complement each other. In acoustical applications, passive systems can usually be efficient only for the attenuation of high-frequency ingredients, whereas $\mathrm{AC}$ is efficient in the range of low frequencies.

The AC problem belongs to the class of inverse source problems and is immediately related to the problems of active noise shielding and active vibration control (see, e.g. Ffowcs Williams, 1984; Nelson \& Elliott, 1992; Fuller et al., 1996). It should be noted here that the existence of the wanted sound component is usually not allowed (Ffowcs Williams, 1984). It seems that Malyuzhinets (1971) was the first who considered the AC problem with the preservation of wanted sound. In application to the Helmholtz equation, he proved the solvability of the problem in unbounded domains, provided that the total field from the primary and secondary sources is known at the perimeter of the protected domain. In a quite general finite-difference formulation, the stationary problem in question was solved by Ryaben'kii (1995) using the apparatus of the difference potential method (DPM) (Ryaben'kii, 2002). Similarly to Malyuzhinets, he assumed the total field from the primary and secondary sources to be known. The obtained generic solution Ryaben'kii (1995) was applied to the Helmholtz equation in 
Lončarić et al. (2001), where it was shown that the controls correspond to a linear combination of single- and double-layer source terms in the continuous formulation. In Ryaben'kii et al. (2007), for the first time the AC problem was formulated and solved for composite domains. In contrast to any previous formulations, in Ryaben'kii et al. (2007) a selective communication between different subdomains was considered. This problem was later analysed in a continuous formulation by Peterson \& Tsynkov (2007). In Ryaben'kii et al. (2009), a variable degree of noise attenuation was considered. The general solution to the stationary AC problem in continuous spaces was obtained by Utyuzhnikov (2009a) via the introduced apparatus of generalized Calderón-Ryaben'kii's (CR) potentials. This solution was extended to non-linear problems in Utyuzhnikov (2010).

For the first time, the non-stationary AC problem in question was tackled in Utyuzhnikov (2009b) where it was proved that the constructed solution is even applicable to resonance regimes. Then, the solution to the problem was obtained in Utyuzhnikov (2009c) via the introduced non-stationary CR potentials. The robustness of the solution was confirmed by the sensitivity analysis carried out in Utyuzhnikov (2009b). The experimental realization of the solution was considered for single and composite domains in Lim et al. (2009) and Lim et al. (2011). In these papers, the AC was realized for pipes with monochromatic and broadband noise being generated in the range up to $1 \mathrm{kHz}$. The achieved noise attenuation approximately corresponded to $30 \mathrm{~dB}$ on average.

Despite a significant progress occurring in this field, practical applications of the obtained solution are quite limited because it does not take into account the reverse effect of the secondary sources in case the wanted sound component is present. As first shown in Ryaben'kii \& Utyuzhnikov (2006), the wanted field is doubled outside the shielded domain. This inevitably affects the input field to be obtained from measurements even in the case of a 'perfect' realization. There have been a few attempts (see Epain \& Friot, 2007; Petersen et al., 2008; Kwon \& Park, 2011) to apply different techniques such as virtual sensing and surface integral control to tackle this problem. All of them are based on trying to predict the wanted sound component and, therefore, are quite limited because the wanted ingredient cannot completely be separated from the total acoustic field. Finally, it is worth noting that the adaptive techniques based on the least means square algorithm are not applicable here since the total sound reduction can damage the wanted sound component which is unknown.

In the current paper, we obtain the solution to the problem in question via the apparatus of CR surface potentials. The key property of these potentials is that they are projections. This allows us to segregate the necessary information from the total field from both primary and secondary sources. On this way, we do not need to predict the wanted sound. The approach can be interpreted as a purely feedforward technique, which requires only the measurement of the total field near the boundary of the protected region. Thus, the effect of the AC should not be measured inside the shielded domain as needed in the feedback techniques (Epain \& Friot, 2007; Petersen et al., 2008; Kwon \& Park, 2011). In contrast to the solution presented in Utyuzhnikov (2009c), the action of the controls must be non-local. This means the operation of each secondary source depends on the total field along the entire boundary surface. The effect of non-locality requires sufficiently quick calculations which can be realized via the DPM. It should be noted that a local solution has recently been obtained in Ryaben'kii (2011) in a finitedifference formulation. However, it requires additional one-dimensional solutions of the wave equation and the implementation of two layers of secondary sources situated on both sides of the boundary of the protected domain. Thus, the prospects of practical realization of the approach suggested in Ryaben'kii (2011) are not clear.

The paper is organized as follows. In Section 2, the general formulation of the problem of AC is given. An introduction to the non-stationary CR potentials is described in Section 3. This apparatus is used in the next section to obtain the solution to the problem in question. Some limitations of the 
obtained solution are also discussed there. Finally, the properties of the solution are outlined in Section 5, in which one-dimensional examples are considered.

\section{General statement of the AC problem}

We consider some bounded domain $D: \bar{D} \subseteq \mathbb{R}^{3}$ with boundary $\Gamma_{0}$ and a subdomain $D^{+}: \bar{D}^{+} \subset D$ with boundary $\Gamma$. Both the boundaries $\Gamma_{0}$ and $\Gamma$ are supposed to be the Lipschitz.

Next, assume that the wave field in the domain $D$ is described by the following linear initialboundary value problem (IBVP):

$$
\begin{aligned}
& L U \stackrel{\text { def }}{=} L_{t} U+L_{y} U=f, \\
& U \in \Xi_{D} .
\end{aligned}
$$

Here, the operators $L_{t}$ and $L_{y}$ are linear differential operators with respect to time and space and $\Xi_{D}$ is the functional space specified below. In particular, the operator $L$ may correspond to the acoustics equations.

We assume that IBVP (1), (2) is well-posed in the sense of Hadamard for any right-hand side $f$ from an appropriate space. The boundary conditions are supposed to be local and implicitly included in the definition of the space $\Xi_{D}$. Without the violation of generality, we assume that the initial conditions are homogeneous. In addition, we suppose that the space $\Xi_{D}$ should not be degenerate. Thus, we assume that the boundary and initial conditions are not over-determined.

We say that a function $U$ is a generalized solution of IBVP (1), (2) if $\langle L U, \Phi\rangle=\langle f, \Phi\rangle$ for any function from some space of test functions. Here, $\langle f, \Phi\rangle$ denotes a linear continuous functional associated with the given generalized function (distribution) $f$. Further, along with a function $U$, we introduce $U_{D^{+}}$ as the restriction of $U$ to $D^{+}$(Vladimirov, 1971).

If the right-hand side in (1) is a regular function, then the IBVP (1), (2) for finding the weak solution is reduced to the following requirement:

$$
\int_{0}^{T} \int_{D}(L U-f, \Phi) \mathrm{d} \mathbf{x} \mathrm{d} t=0
$$

for any basic (test) function $\Phi \in C_{0}^{\infty}\left(K_{T}\right)$, where $(\cdot, \cdot)$ means a scalar product, $K_{T}:=D \times(0, T)(T>0)$.

The wave sources, represented by the right-hand side $f$, are assumed to be placed both on $D^{+}$and outside $D^{+}$(see Fig. 1):

$$
\begin{aligned}
& f=f^{+}+f^{-}, \\
& \operatorname{supp} f^{+} \subset D^{+}, \\
& \operatorname{supp} f^{-} \subset D^{-} \stackrel{\text { def }}{=} D \backslash \bar{D}^{+} .
\end{aligned}
$$

We interpret $f^{+}$as wanted sources, while $f^{-}$corresponds to unwanted (noise) sources.

Next, suppose that in (1) the right-hand side $f \in F_{D}$ where the space $F_{D}$ is such that the solution to IBVP (1), (2) exists. Thus, spaces $\Xi_{D}$ and $F_{D}$ are isomorphic to each other. Hereafter, we assume: $f \in F_{D} \Longrightarrow \theta\left(D^{+}\right) f \in F_{D}$, where $\theta\left(D^{+}\right)$is the characteristic function of $D^{+}$equal to 1 on $D^{+}$and 0 outside. Obviously, this assumption is supposed to be valid for the AC problem since $f^{+} \in F_{D}$.

In the AC problem, we protect the domain $D$ from the field generated by sources $f^{-}$situated in $D^{-}$. Then we arrive at the following inverse source problem: find additional sources $G$ in $\bar{D}^{-}$such that the 


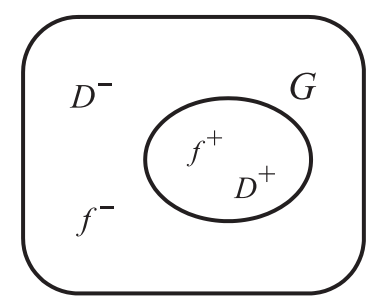

FIG. 1. Domain sketch.

solution to the IBVP

$$
\begin{aligned}
& L W=f+G, \\
& \operatorname{supp} G \subset \bar{D}^{-}, \\
& W \in \Xi_{D}
\end{aligned}
$$

coincides on the domain $D^{+}$with the solution to $\operatorname{IBVP}(1),(2)$ if $f^{-} \equiv 0$ :

$$
\begin{aligned}
& L U^{+}=f^{+}, \\
& U^{+} \in \Xi_{D} .
\end{aligned}
$$

Thus, it is required that the functions $U$ and $W$ be identical on the domain $D^{+}: W_{D^{+}}=U_{D^{+}}^{+}$.

Further, we introduce an additional requirement such that the control has to be distributed on the boundary $\Gamma$ : supp $G \subset \Gamma$. This requirement can be important for practical applications.

Our key assumption on the input data of the AC problem is that the field $W$ is only known in the vicinity of the boundary $\Gamma$.

The general solution to the AC problem, formulated above, is based on the theory of potentials described in the next section.

\section{Non-stationary $\mathrm{CR}$ potentials}

Following Utyuzhnikov (2009c), we introduce an operator $P_{D^{+}}: \Xi_{D^{+}} \rightarrow \Xi_{D^{+}}, \Xi_{D^{+}}=\left\{U_{D^{+}} \mid U \in \Xi_{D}\right\}$, as follows:

\section{DEFINITION 3.1}

$$
P_{D^{+}} V_{D^{+}} \stackrel{\text { def }}{=} L_{D^{+}}^{-1}\left(\theta\left(\bar{D}^{-}\right) L V\right)
$$

where $L_{D^{+}}^{-1} g \stackrel{\text { def }}{=} L^{-1} g_{\mid D^{+}}, \theta\left(\bar{D}^{-}\right)$is the characteristic function of $\bar{D}^{-}$.

It is worth noting that, since the IBVP is well-posed, the inverse operator $L^{-1}$ in Definition 3.1 is defined .

The authors of Ryaben'kii (2002) and Utyuzhnikov (2009a) introduced the notion of a clear trace $\operatorname{Tr}^{+}(\Gamma) U_{D}$, assigned to the operator $P_{D^{+}}$, which can be defined as

$$
\operatorname{Tr}^{+}(\Gamma) V_{D^{+}}=\operatorname{Tr}^{+}(\Gamma) W_{D^{+}} \Rightarrow P_{D^{+}} V_{D^{+}}=P_{D^{+}} W_{D^{+}}
$$


Here, $\operatorname{Tr}^{+}(\Gamma)$ is a boundary operator: $\Xi_{D^{+}} \rightarrow \Xi_{\Gamma}, \Xi_{\Gamma} \subset \oplus_{j=0}^{k-1} H^{s-1 / 2-j}(\Gamma)$, where $k$ is the order of the operator $L_{y}, s>k-1 / 2$.

Thus, the volume potential $P_{D^{+}} V_{D^{+}}$is fully determined by the clear trace of $V_{D^{+}}$. Then, we arrive at the definition of a surface potential $P_{D^{+} \Gamma}: \Xi_{\Gamma} \rightarrow \Xi_{D^{+}}$with density $\xi_{\Gamma}$.

DEFINITION 3.2

$$
P_{D^{+} \Gamma} \xi_{\Gamma} \stackrel{\text { def }}{=} P_{D^{+}} V_{D^{+}},
$$

where $\xi_{\Gamma}=\operatorname{Tr}^{+}(\Gamma) V$.

As proved in Utyuzhnikov (2009c), Definition 3.2 is correct. This means the value of the potential $P_{D^{+} \Gamma} \xi_{\Gamma}$ is completely determined by its density $\xi_{\Gamma}$. In addition, it is to be noted that the potential $P_{D^{+} \Gamma} \xi_{\Gamma}$ is a retarded surface potential because it is based on the solution of an IBVP and implicitly includes a retarded time.

Next, we obtain the clear trace associated with the operator $L$. For this purpose, let us introduce a trace operation as follows. Let $\Gamma_{\epsilon}^{+}$be smooth manifolds parallel to $\Gamma$ in the sense of Lions \& Magenes (1972, Chapter 2): $\Gamma_{\epsilon}^{+} \subset D^{+}, \Gamma_{\epsilon}^{+} \rightarrow \Gamma$ if $\epsilon \rightarrow 0$. Then, the trace operator $\operatorname{Tr}_{\Gamma}^{+}: H^{s}\left(D^{+}\right) \rightarrow H^{s-1 / 2}(\Gamma)$ is given by

$$
\operatorname{Tr}_{\Gamma}^{+} U_{D^{+}} \stackrel{\text { def }}{=} \lim _{\epsilon \rightarrow 0} \operatorname{Tr}_{\Gamma_{\epsilon}^{+}} U_{D^{+}}
$$

where

$$
\operatorname{Tr}_{\Gamma_{\epsilon}^{+}} U_{D^{+}} \stackrel{\text { def }}{=} U_{D^{+}}(\mathbf{x}), \quad \mathbf{x} \in \Gamma_{\epsilon}^{+}
$$

One can similarly introduce the operator $\operatorname{Tr}_{\Gamma}^{-}: H_{0}^{s}\left(D^{-}\right) \rightarrow H^{s-1 / 2}(\Gamma)$.

It is worth noting that the operator $\operatorname{Tr}^{+}(\Gamma)$ in definition (7) of the clear trace often coincides with the Cauchy data trace operator of the operator $L_{y}$ :

$$
\begin{aligned}
& \operatorname{Tr}_{c}^{+}(\Gamma): \Xi_{D^{0}} \rightarrow \pi_{c}(\Gamma) \subset \oplus_{0}^{k-1} H^{s-1 / 2-j}(\Gamma), \\
& \operatorname{Tr}_{c}^{+}(\Gamma) U \stackrel{\text { def }}{=} \operatorname{Tr}_{\Gamma}^{+}\left(U, \frac{\partial U}{\partial \mathbf{n}}, \ldots, \frac{\partial^{k-1} U}{\partial \mathbf{n}^{k-1}}\right)^{\top},
\end{aligned}
$$

where $k$ is the order of the operator $L$ and $\mathbf{n}$ is the external normal vector to the boundary $\Gamma$. Here, the term a normal derivative refers to the regular normal derivative (Vladimirov, 1971). Operator $\operatorname{Tr}_{c}^{-}(\Gamma)$ : $\Xi_{D^{0}} \rightarrow \pi_{c}(\Gamma)$ is determined in a similar way.

Next, consider first- and second-order spatial differential operators $L_{y}$.

We represent the first-order operator $L_{y}$ by

$$
L_{y}:=L_{f} \stackrel{\text { def }}{=} \sum_{1}^{3} A^{i} \frac{\partial}{\partial y^{i}},
$$

where $\left\{y^{i}\right\}(i=1,2,3)$ is the Cartesian coordinate system; $A^{i}$ are $n \times n$ matrices: $A^{i}=A^{i}(\mathbf{y}) \in C^{1}(\bar{D})$. 
The second-order operator is given by the following elliptic operator:

$$
L_{y}:=L_{s} \stackrel{\text { def }}{=}-\nabla(p \nabla)-q,
$$

where $p \in C^{1}(\bar{D}), q \in C(\bar{D})$ and $p>0$.

As shown in Utyuzhnikov (2009c),

$$
P_{D^{+} \Gamma} \xi_{\Gamma}=-L_{D^{+}}^{-1}\left(A_{\Gamma} \zeta\left(\xi_{\Gamma}\right)\right)
$$

where $\xi_{\Gamma} \stackrel{\text { def }}{=} \operatorname{Tr}^{+}(\Gamma) V=\operatorname{Tr}_{\Gamma}^{+} V_{D^{+}}, V \in \Xi_{D}$. In addition:

- if $L_{y}:=L_{f}$, then $\xi_{\Gamma}=V_{\Gamma}, \zeta\left(\xi_{\Gamma}\right)=V_{\Gamma} \delta(\Gamma)$ and $A_{\Gamma}=A_{n}$, where $\mathbf{n}$ is the outward normal to the boundary $\Gamma$ and $\delta(\Gamma)$ is the surface delta function;

- if $L_{y}:=L_{s}$, then $\xi_{\Gamma}=\left(V_{\Gamma},(\partial / \partial \mathbf{n}) V\right)^{\top}, \zeta\left(\xi_{\Gamma}\right)=\left(V_{\Gamma}(\partial / \partial \mathbf{n}) \delta(\Gamma),(\partial / \partial \mathbf{n}) V \delta(\Gamma)\right)^{\top}$ and $A_{\Gamma}=$ $-p_{\Gamma}(1,1)$.

In the case of $L_{S}$, the potential $P_{D^{+}} \xi_{\Gamma}$ is represented by a linear combination of single- and doublelayer potentials:

$$
P_{D^{+} \Gamma} \xi_{\Gamma}=L_{D^{+}}^{-1}\left(p_{\Gamma} \xi_{\Gamma}^{(2)} \delta(\Gamma)+\frac{\partial}{\partial \mathbf{n}}\left(p_{\Gamma} \xi_{\Gamma}^{(1)} \delta(\Gamma)\right)\right)
$$

where $\xi_{\Gamma}=\left(\xi_{\Gamma}^{(1)}, \xi_{\Gamma}^{(2)}\right)^{\top} \equiv \operatorname{Tr}_{\Gamma}^{+}\left(V_{D^{+}},(\partial / \partial \mathbf{n}) V_{D^{+}}\right)^{\top}, V \in \Xi_{D}$.

In a general case, Equation (12) can be rewritten as follows:

$$
P_{D^{+} \Gamma} \operatorname{Tr}^{+}(\Gamma) V=L_{D^{+}}^{-1}\left(\theta_{\bar{D}^{-}} L_{y}\left(\theta_{D^{+}} V\right)\right) .
$$

As proved in Utyuzhnikov (2009c), the non-stationary CR potential is a projection. This key property of the CR potentials means

$$
P_{D^{+}} U_{D^{+}}^{+}=0_{D^{+}}
$$

and

$$
P_{D^{+}} U_{D^{+}}^{-}=U_{D^{+}}^{-}
$$

Properties (14) and (15) were first obtained in the stationary formulation in Ryaben'kii (2002) and Utyuzhnikov (2009a).

Thus, the field generated in $D^{+}$does not contribute to the potential, while the field generated outside $D^{+}$is projected by the operator $P_{D^{+}}$onto itself. Hence, the operator $P_{D^{+}}$is a projection.

In addition, the operator $P_{D^{+} \Gamma}$ is also a projection and able to separate the unwanted ingredient:

$$
P_{D^{+} \Gamma} \operatorname{Tr}^{+}(\Gamma) U_{D^{+}}=U_{D^{+}}^{-} .
$$

Property (16) immediately follows from the linearity of the problem, Definition (3.2), and equalities (14) and (15). It is worth noting here that properties (14-16) are even valid in the non-linear case as have recently been proved in Utyuzhnikov (2010).

\section{Real-time control}

On the basis of the potential introduced in the previous section, one can obtain the solution to the AC problem formulated in Section 2. 
As shown in Utyuzhnikov (2009c), if the total field from the primary sources is known, then the control can be provided by the secondary source:

$$
G_{0}=A_{\Gamma} \zeta\left(\operatorname{Tr}^{+}(\Gamma) U\right)
$$

where $A_{\Gamma}$ and $\zeta=\zeta\left(\xi_{\Gamma}\right)$ are determined in Equation (12) or

$$
G_{0}=-\theta_{\bar{D}^{-}} L_{y}\left(\theta_{D^{+}} U\right)
$$

In Ryaben'kii et al. (2007), it was demonstrated that solution (17) allows us to protect the domain $D$ from the field generated outside, with the entire wanted field being preserved. In particular, the wanted field reflected from the boundaries is also retained. The drawback of the solution (17) is that it is based on the $\operatorname{Tr}^{+}(\Gamma) U$. Practically, it is to be measured near the boundary $\Gamma$. However, as shown in Utyuzhnikov (2009c), the wanted sound is doubled outside the domain $D$ due to control (17). Thus, it affects the input information which no longer represents the total field from the primary sources required in (17). Hence, the control (17) can be used either without wanted sound or with the assumption that the field from the primary sources is known. The latter case was realized in Lim et al. (2011) where the required primary field was recorded in advance and then used in a repeated experiment. It is clear that the practical applications of the solution (17) are quite limited.

Now, assume that the total field $W$ from both the primary and secondary sources is known near the boundary $\Gamma$. It is clear that the function $W$ must have a jump across the boundary $\Gamma$ due to control (17). More precisely, we assume that the field $W$ is known on the external side of the boundary $\Gamma$ with respect to the domain $D$. Then, from the projection property of the potential (16) we have

$$
P_{D^{+} \Gamma} \operatorname{Tr}_{c}^{-}(\Gamma) W=U_{D^{+}}^{-} .
$$

Thus, the potential operator $P_{D^{+} \Gamma} \operatorname{Tr}_{c}^{-}(\Gamma)$ filters the contribution of the control. Then, we can apply solution (17) to the unwanted field. Hence, the AC source is given by

$$
G_{0}=A_{\Gamma} \zeta\left(P_{\Gamma} \operatorname{Tr}_{c}^{-}(\Gamma) W\right)
$$

where $P_{\Gamma}:=\operatorname{Tr}^{+}(\Gamma) P_{D^{+} \Gamma}$.

In terms of the operator $L$, the control reads

$$
G_{0}=-\theta_{\bar{D}^{-}} L_{y}\left(\theta_{D^{+}} P_{D^{+} \Gamma} \operatorname{Tr}_{c}^{-}(\Gamma) W\right) .
$$

Thus, the measurements must be carried out on the external side of the boundary $\Gamma$. The control (19) is non-local due to the operator $P_{D^{+} \Gamma}$. Hence, the realization of AC (19) requires the solution of an IBVP in the domain $D$.

It is worth noting that $P_{\Gamma} \operatorname{Tr}_{c}^{-}(\Gamma) U^{+}=0_{\Gamma}$. Thus, the control (19) efficiently filters the wanted sound while retaining the noise ingredient to be attenuated.

\subsection{Limitations}

The application of the obtained solution requires the calculation of the potential $P_{D^{+} \Gamma} \xi_{\Gamma}$. Its value depends on the boundary conditions at the boundary $\Gamma_{0}$ which can be unknown. To overcome this problem, we can calculate the potentials with specified boundary conditions. For example, we can set non-reflecting boundary conditions. These boundary conditions can be obtained as a transfer of the 
Sommerfeld boundary conditions from infinity to the boundary $\Gamma_{0}$ (Tsynkov, 1998). In this case, only the reflected component of the wanted field may be lost due to the AC. It is clear that, for many practical applications, this ingredient is not important. Moreover, we can specify boundary conditions which can imitate the acoustic medium with wanted properties. For example, if we protect the compartment of a car, we can improve acoustics via imitation of specific boundary conditions which can be prescribed in advance in the calculation of the potentials. It is to be noted that, regardless of the boundary conditions, the unwanted ingredient cannot penetrate into the protected domain because the operator $P_{\Gamma}$ is a projector.

The CR potentials have to be calculated in a real-time regime. Therefore, the algorithm is only applicable to low frequency noise. There are also very strict requirements to the computational efficiency of the algorithm used. In this way, the DPM provides an efficient technique based on the spectral approach (Ryaben'kii, 2002). The operation of the controls must be calculated while the signal propagates from the sensors to the loudspeakers. Thus, some displacement between the level of sensors and level of controls is necessary.

The practical realization of the algorithm requires the measurement of the sound pressure and normal component of the particle velocity along the entire boundary of the protected region. It can only be done at a finite number of places. The same limitation is applicable to the controls. This inevitably leads to some error.

Next, consider the applications of solution (17) to one-dimensional problems. In all examples, we assume that the boundary conditions are set at infinity in such a way that the solution is unique. For example, we can specify the Sommerfeld boundary conditions used in acoustics. The initial conditions are supposed to be homogeneous.

\section{Examples}

\subsection{Wave equation}

As an illustration of the results obtained in the previous section, assume that $L$ coincides with the operator of the wave equation:

$$
L:=\frac{\partial^{2}}{\partial t^{2}}-a^{2} \Delta,
$$

where $\Delta$ is the Laplace operator and $a$ is a constant corresponding to the speed of sound.

Suppose that the initial conditions are homogeneous:

$$
U(\mathbf{x}, 0)=0, \quad U_{t}(\mathbf{x}, 0)=0 .
$$

In addition, we assume that domain $D$ corresponds to space $\mathbb{R}^{3}$. Then, the free-space Green's function, Gr, is well known and given by Vladimirov (1971):

$$
\mathrm{Gr}=\frac{\delta(t-|\mathbf{x}| / a)}{4 \pi a^{2}|\mathbf{x}|}
$$

The solution to IBVP (1), (2) is given by

$$
U(\mathbf{x})=\int_{\mathbb{R}^{3}, \tau} \operatorname{Grf} \mathrm{d} \mathbf{y} \mathrm{d} \tau, \quad \mathbf{x} \in \mathbb{R}^{3} .
$$


Here and further in this section, the integral contains convolutions over indicated space and past time.

On the other hand, the solution can be represented by Kirchhoff's formula (Morse \& Feshbach, 1953; Laliena \& Sayas, 2009):

$$
U(\mathbf{x})=\int_{D^{+}, \tau} \operatorname{Gr} f \mathrm{~d} y \mathrm{~d} \tau+a^{2} \int_{\Gamma, \tau}\left(\operatorname{Gr} U_{n}-U \mathrm{Gr}_{n}\right) \mathrm{d} \sigma \mathrm{d} \tau, \quad \mathbf{x} \in D^{+} .
$$

Similar results are applicable to the functions $U^{+}$and $U^{-}$.

Thus, we have that, in $D^{+}$,

$$
U^{+}(\mathbf{x})=\int_{D^{+}, \tau} \operatorname{Gr} f^{+} \mathrm{d} y \mathrm{~d} \tau+a^{2} \int_{\Gamma, \tau}\left(\operatorname{Gr} U_{n}^{+}-U^{+} \mathrm{Gr}_{n}\right) \mathrm{d} \sigma \mathrm{d} \tau, \quad \mathbf{x} \in D^{+} .
$$

Since $\operatorname{supp} f^{+} \subset D^{+}$, we obtain

$$
\int_{\Gamma, \tau}\left(\operatorname{Gr} U_{n}^{+}-U^{+} \mathrm{Gr}_{n}\right) \mathrm{d} \sigma \mathrm{d} \tau=0, \quad \mathbf{x} \in D^{+}
$$

In turn, in $D^{-}:=\mathbb{R}^{3} \backslash \bar{D}^{+}$we have

$$
U^{+}(\mathbf{x})=-a^{2} \int_{\Gamma, \tau}\left(\operatorname{Gr} U_{n}^{+}-U^{+} \mathrm{Gr}_{n}\right) \mathrm{d} \sigma \mathrm{d} \tau, \quad \mathbf{x} \in D^{-} .
$$

In a similar way, we can obtain that, in $D^{+}$,

$$
U^{-}(\mathbf{x})=a^{2} \int_{\mathbb{R}^{3}, \tau}\left(\mathrm{Gr} U_{n}^{-}-U^{-} \mathrm{Gr}_{n}\right) \mathrm{d} \mathbf{y} \mathrm{d} \tau, \quad \mathbf{x} \in D^{+} .
$$

Now, consider a surface source $G_{0}$ :

$$
G_{0}=-a^{2} U_{n \mid \Gamma} \delta(\Gamma)-a^{2}\left(U_{\Gamma} \delta(\Gamma)\right)_{n} .
$$

Then, the sound field generated by this source in $D^{+}$is represented by an appropriate combination of single- and double-layer potentials:

$$
V(\mathbf{x})=\int_{\mathbb{R}^{3}, \tau} \operatorname{Gr} G_{0} \mathrm{~d} \mathbf{y} \mathrm{d} \tau=-a^{2} \int_{\Gamma, \tau}\left(\operatorname{Gr} U_{n}-U \mathrm{Gr}_{n}\right) \mathrm{d} \sigma \mathrm{d} \tau=-U^{-}(\mathbf{x}), \quad \mathbf{x} \in D^{+} .
$$

Thus, $V$ is the annihilating field and the control based on the total field $U$ from the primary sources is capable of attenuating the unwanted sound.

On the other hand, in $D^{-}$the function $V$ has the following value:

$$
V(\mathbf{x})=-a^{2} \int_{\Gamma, \tau}\left(\operatorname{Gr} U_{n}-U \mathrm{Gr}_{n}\right) \mathrm{d} \sigma \mathrm{d} \tau=U^{+}(\mathbf{x}), \quad \mathbf{x} \in D^{-} .
$$

Thus, the contribution of the control outside domain $D^{+}$coincides with the wanted sound. Hence, the wanted sound outside $D^{+}$is doubled. Therefore, if the wanted sound exists, the control inevitably affects any measurement outside $D^{+}$. Hence, this makes it impossible to measure the total field $U$ from the primary sources since the wanted sound is unknown. 
In terms of the AC represented by (19), the algorithm is the following. The control is situated at the surface $\Gamma$. The input (reference) signal is measured on surface $\Gamma_{\epsilon}^{-}: \Gamma_{\epsilon}^{-} \subset D^{-}, \Gamma_{\epsilon}^{-} \rightarrow \Gamma$ if $\epsilon \rightarrow 0$. Thereby, the surface $\Gamma_{\epsilon}^{-}$bounds some domain $D_{\epsilon}^{+}: D^{+} \subset D_{\epsilon}^{+}, \partial D_{\epsilon}^{+}:=\Gamma_{\epsilon}^{-}$.

Next, let the input field be $W_{\Gamma_{\epsilon}^{-}}$and $W_{n \mid \Gamma_{\epsilon}^{-}}$. Then, we can calculate $U^{-}$and $U_{n}^{-}$at $\Gamma$ :

$$
U^{-}(\mathbf{x})=a^{2} \int_{\Gamma_{\epsilon}^{-}, \tau}\left(\operatorname{Gr} W_{n}-W \mathrm{Gr}_{n}\right) \mathrm{d} \sigma \mathrm{d} \tau, \quad \mathbf{x} \in D_{\epsilon}^{+} .
$$

As soon as $U_{\Gamma}^{-}$and $U_{n \mid \Gamma}^{-}$are known, the control can be obtained straightforwardly:

$$
G_{0}=-a^{2} U_{n \mid \Gamma}^{-} \delta(\Gamma)-a^{2}\left(U_{\Gamma}^{-} \delta(\Gamma)\right)_{n}
$$

Thus, if the wanted sound component exists, real-time control provided by (17) is not applicable. This means the control cannot be local and requires taking into account the field over the boundary $\Gamma$. In the example in question, Green's function is supposed to be known and the control can be calculated from (23) and (24). Their combination is equivalent to the use of control (19). One can see the control (23), (24) is based on the integral over $\Gamma$ and past time. At each point of control, the effect of other boundary points automatically takes into account retarded time.

Finally, it is important to note the control (19) is more general because it does not require the knowledge of Green's function.

\subsection{Semi-space}

First, consider the transport equation

$$
u_{t}+a u_{x}=f
$$

where $a$ is a constant.

Assume that we shield semi-space $\mathbb{R}^{-}: x<0$ from noise generated in semi-space $\mathbb{R}^{+}: x>0$.

If $a>0$, then we can put any source at $x=0$ without any effect for the shielded domain. Now consider $a<0$ and assume that there is only noise source in $\mathbb{R}^{+}: \operatorname{supp} f \subset \mathbb{R}^{+}$. Then, control source (17) is represented by $s_{0}=a u(0, t) \delta(x)$.

One can see that the solution to equation

$$
\bar{u}_{t}+a \bar{u}_{x}=f+s_{0}
$$

is given by $\bar{u}=\theta(x) u$.

Instead of $s_{0}$, we can introduce control (19): $\bar{s}_{0}=\bar{u}(+0, t) \delta(x)$, where $x=+0$ means the value of $\bar{u}$ at the right-hand side of the point source placed at $x=0$. Since $a$ is negative, the control $\bar{s}_{0}$ does not affect the measurement. Thus, $\bar{u}(+0, t)=u(0, t)$ and $\bar{s}_{0}=s_{0}$.

It is clear that the control $\bar{s}_{0}$ is also applicable if there are wanted sources in $\mathbb{R}^{-}$since they do not contribute to $\bar{u}(+0, t)$ if $a<0$.

Consider now the Euler acoustics equations:

$$
\begin{aligned}
& \frac{\bar{p}_{t}}{\rho_{0} c_{0}^{2}}+\bar{v}_{x}=\frac{1}{\rho_{0} c_{0}^{2}} f^{(p)}+q_{\mathrm{vol}}, \\
& \rho_{0} \bar{v}_{t}+\bar{p}_{x}=f^{(v)}+f_{\mathrm{vol}},
\end{aligned}
$$


where $\bar{v}$ is the particle velocity; $\bar{p}$, sound pressure; $f^{(p)}$ and $f^{(v)}$, acoustic sources; $q_{\mathrm{vol}}$ and $f_{\mathrm{vol}}$ are controls; parameters marked by 0 are constant.

From (17) it follows that the controls are represented by

$$
\begin{aligned}
q_{\mathrm{vol}} & =\bar{v}(+0, t) \delta(x), \\
f_{\mathrm{vol}} & =\bar{p}(+0, t) \delta(x) .
\end{aligned}
$$

To demonstrate the applicability of the control (28), we can rewrite the set (27) in the Riemann invariants:

$$
\begin{aligned}
& R_{t}^{+}+c_{0} R_{x}^{+}=c_{0} R^{+} \delta(x)+\frac{f^{(v)}}{\rho_{0}}+\frac{f^{(p)}}{\rho_{0} c_{0}}, \\
& R_{t}^{-}-c_{0} R_{x}^{-}=-c_{0} R^{-} \delta(x)+\frac{f^{(v)}}{\rho_{0}}-\frac{f^{(p)}}{\rho_{0} c_{0}},
\end{aligned}
$$

where $R^{+}=\bar{u}+\bar{p} / \rho_{0} c_{0}, R^{-}=\bar{u}-\bar{p} / \rho_{0} c_{0}$.

From the previous analysis of the transport equation, it immediately follows that semi-space $\mathbb{R}^{-}: x<0$ is shielded by controls (28).

Next, eliminating $\bar{p}$ from set (27), we can obtain the AC for the wave equation:

$$
\bar{u}_{t t}-c_{0}^{2} \bar{u}_{x x}=f_{w}+c_{0}^{2} s_{0}
$$

where $f_{w}=(1 / \rho)\left(f_{t}^{(v)}-f_{x}^{(p)}\right)$,

$$
s_{0}=-\bar{u}_{x}(+0, t) \delta(x)-(\bar{u}(+0, t) \delta(x))_{x}
$$

One can see that control (31) corresponds to the general solution (17).

Thus, control (17) is applicable to the AC problem in the semi-space although this domain is not bounded.

\subsection{Bounded domain}

In the case of a bounded domain, the AC proves to be non-local.

First, consider the potential in interval $\left[x_{A}, x_{B}\right]$. According to (13), the potential with density $\xi_{\Gamma}=$ $\left(\xi_{1 A}, \xi_{2 A}, \xi_{1 B}, \xi_{2 B}\right)^{\top}$ can be calculated as follows:

$$
w_{t t}-c_{0}^{2} w_{x x}=c_{0}^{2}\left(g_{B}-g_{A}\right),
$$

where

$$
\begin{aligned}
& g_{B}=\xi_{2 B} \delta\left(x-x_{B}\right)+\left(\xi_{1 B} \delta\left(x-x_{B}\right)\right)_{x}, \\
& g_{A}=-\xi_{2 A} \delta\left(x-x_{A}\right)+\left(\xi_{1 A} \delta\left(x-x_{A}\right)\right)_{x},
\end{aligned}
$$

Then, $P_{A B} \xi_{\Gamma}=w(x), x \in\left[x_{A}, x_{B}\right]$. 
Then, the algorithm of the $\mathrm{AC}$ is the following. First, we solve an auxiliary problem (it is to be solved in real time):

$$
v_{t t}-c_{0}^{2} v_{x x}=c_{0}^{2}\left(s_{B}-s_{A}\right)
$$

where

$$
\begin{aligned}
& s_{B}=\bar{u}_{x}\left(x_{B}+0, t\right) \delta\left(x-x_{B}\right)+\left(\bar{u}\left(x_{B}+0, t\right) \delta\left(x-x_{B}\right)\right)_{x}, \\
& s_{A}=\bar{u}_{x}\left(x_{A}-0, t\right) \delta\left(x-x_{A}\right)+\left(\bar{u}\left(x_{A}-0, t\right) \delta\left(x-x_{A}\right)\right)_{x} .
\end{aligned}
$$

Here, 'bar' corresponds to the total field from both primary and secondary sources.

The solution to the auxiliary problem allows us to extract noise from the total field. Then, we obtain the following controls:

$$
\begin{aligned}
& G_{B}=v_{x}\left(x_{B}-0, t\right) \delta\left(x-x_{B}\right)+\left(v\left(x_{B}-0, t\right) \delta\left(x-x_{B}\right)\right)_{x}, \\
& G_{A}=v_{x}\left(x_{A}+0, t\right) \delta\left(x-x_{A}\right)+\left(v\left(x_{A}+0, t\right) \delta\left(x-x_{A}\right)\right)_{x} .
\end{aligned}
$$

The final equation reads

$$
\bar{u}_{t t}-c_{0}^{2} \bar{u}_{x x}=f_{w}-c_{0}^{2}\left(G_{B}-G_{A}\right) .
$$

It is clear that the operation of each control $G_{A}$ and $G_{B}$ depends on the field at both boundary points $A$ and $B$. Thus, the control is non-local. Practically, the controls at points $A$ and $B$ should operate on the data obtained at the same time moment. It is realistic if they are connected in a network. In such a case, the information between the controls propagates with the speed of light.

One can note that in the experiments considered in Lim et al. (2009, 2011), the local control (17) was used. This required repetition of the experiments in the case of the presence of a wanted sound component. The control (19), which is described in this section, provides an opportunity to carry out the experiments from Lim et al. $(2009,2011)$ in a real-time regime.

\section{Conclusion}

The general solution to a non-stationary AC problem has been obtained using the theory of the CR potentials. In contrast to many other approaches, it allows the wanted component of sound to exist. The principal novelty of the approach is that it can be realized in a real-time regime even in case a wanted sound component is present. As the result, the control must be non-local in such a way that the operation of each secondary source depends on the measurements to be carried out along the entire boundary.

\section{Acknowledgement}

The author is grateful to Professor Victor S. Ryaben'kii for fruitful discussions and the unknown referees for useful remarks.

\section{REFERENCES}

Epain, N. \& FRIOT, E. (2007) Active control of sound inside a sphere via control of the acoustic pressure at the boundary surface. J. Sound and Vibration, 299, 587-604.

Ffowcs Williams, J. E. (1984) Review lecture: anti-sound. Proc. R. Soc. Lond. Ser. A. Math. Phys. Sci., 395, 63-88.

Fuller, C. R., Nelson, P. A. \& Elliott, S. J. (1996) Active Control of Vibration. London: Academic Press. 
KwON, B. \& PARK, Y. (2011) Active window based on the prediction of interior sound field: experiment for a band-limited noise. Inter-Noise 2011, Osaka, Japan, September 4-7.

Laliena, A. \& Sayas, F. J. (2009) A distributional version of Kirchhoff's formula. J. Math. Anal. Appl., 359, 197-208.

Lim, H., Utyuzhnikov, S. V., Lam, Y. W., Turan, A., Avis, M. R., Ryaben'kiI, V. S. \& Tsynkov, S. V. (2009) An experimental validation of the active noise control methodology based on difference potentials. AIAA J., 47, 874-884.

Lim, H., Utyuzhnikov, S. V., Lam, Y. W. \& Turan, A. (2011) Multi-domain active sound control and noise shielding. J. Acoust. Soc. Amer., 129, 717-725.

Lions, J. L. \& Magenes, E. (1972) Non-Homogeneous Boundary Value Problems and Applications. BerlinHeidelberg-New York: Springer.

LonČarić, J., Ryaben'KiI, V. S. \& Tsynkov, S. V. (2001) Active shielding and control of noise. SIAM J. Appl. Math., 62, 563-596.

MaLYUZHINETS, G. D. (1971) An unsteady diffraction problem for the wave equation with compactly supported right-hand side. Proceeding of the Acoustics Institute, USSR Academic Science, Moscow. pp. 124-139 (in Russian).

Morse, P. M. \& Feshbach, H. (1953) Methods of Theoretical Physics, Vol. I, Chapter 7. New York: McGraw-Hill Book Company Inc.

Nelson, P. A. \& Elliott, S. J. (1992) Active Control of Sound. San Diego, CA: Academic Press.

Petersen, C. D., Froonje, R., Cazzolato, B. S., Zander, A. C. \& Hansen, C. H. (2008) A Kalman filter approach to virtual sensing for active noise control. Mech. Syst. Signal Process., 22, 490-508.

Peterson, A. \& Tsynkov, S. V. (2007) Active control of sound for composite regions. SIAM J. Appl. Math., 67, $1582-1609$.

RyABEN'KII, V. S. (1995) A difference shielding problem. Funct. Anal. Appl., 29, 70-71.

RYABEN'KII, V. S. (2002) Method of Difference Potentials and its Applications. Springer: Berlin.

RYABEN'KII, V. S. (2011) Model of real-time active noise shielding of a given subdomain subject to external noise sources. J. Comput. Math. Math. Phys., 51, 444-454.

Ryaben'ki, V. S., Tsynkov, S. V. \& Utyuzhnikov, S. V. (2007) Inverse source problem and active shielding for composite domains. J. Appl. Math. Lett., 20, 511-515.

Ryaben'KiI, V. S., Tsynkov, S. V. \& UtyuZhnikov, S. V. (2009) Active control of sound with variable degree of cancellation. J. Appl. Math. Lett., 22, 1846-1851.

RyABen'KII, V. S. \& UtYUZhNiKov, S. V. (2006) Active shielding model for hyperbolic equations. IMA J. Appl. Math., 71, 924-939.

Ryaben'KiI, V. S., Utyuzhnikov, S. V. \& Turan, A. A. (2007) On the application of difference potential theory to active noise control. J. Adv. Appl. Math., 20, 511-515.

Tsynkov, S. V. (1998) Numerical solution of problems on unbounded domains. A review. J. Appl. Numer. Math., 27, 465-532.

Utyuzhnikov, S. V. (2009a) Generalized Calderón-Ryaben'kii potentials. IMA J. Appl. Math., 74, 128-148.

Utyuzhnikov, S. V. (2009b) Non-stationary problem of active sound control in bounded domains. J. Comput. Appl. Math., 43, 101-112.

Utyuzhnikov, S. V. (2009c) Active wave control and generalized surface potentials. J. Adv. Appl. Math., 43, $101-112$.

Utyuzhnikov, S. V. (2010) Nonlinear problem of active sound control. J. Comput. Appl. Math., 234, 215-223.

VLadimirov, V. S. (1971) Equations of Mathematical Physics. New York: Dekker. 